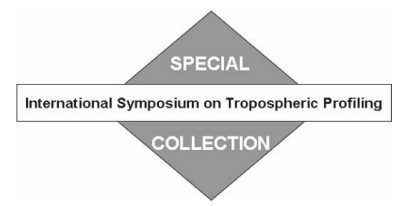

\title{
Effects of Wind Field Inhomogeneities on Doppler Beam Swinging Revealed by an Imaging Radar
}

\author{
B. L. Cheong, ${ }^{*}$ T.-Y. Yu, ${ }^{+}$R. D. Palmer, ${ }^{*}$ K.-F. Yang, ${ }^{\#}$ M. W. Hoffman, ${ }^{\circledR}$ S. J. Frasier, ${ }^{\&}$ And \\ F. J. LOPEZ-DEKKER \& \\ * School of Meteorology, University of Oklahoma, Norman, Oklahoma \\ + School of Electrical and Computer Engineering, University of Oklahoma, Norman, Oklahoma \\ \# Institute of Space Science, National Central University, Jhongli, Taiwan \\ @ Department of Electrical Engineering, University of Nebraska, Lincoln, Nebraska \\ \& Department of Computer and Electrical Engineering, University of Massachusetts-Amherst, Amherst, Massachusetts
}

(Manuscript received 9 January 2007, in final form 20 August 2007)

\begin{abstract}
In this work, the accuracy of the Doppler beam-swinging (DBS) technique for wind measurements is studied using an imaging radar-the turbulent eddy profiler (TEP) developed by the University of Massachusetts, with data collected in summer 2003. With up to 64 independent receivers, and using coherent radar imaging (CRI), several hundred partially independent beams can be formed simultaneously within the volume defined by the transmit beam. By selecting a subset of these beams, an unprecedented number of DBS configurations with varying zenith angle, azimuth angle, and number of beams can be investigated. The angular distributions of echo power and radial velocity obtained by CRI provide a unique opportunity to validate the inherent assumption in the DBS method of homogeneity across the region defined by the beam directions. Through comparison with a reference wind field, calculated as the optimal uniform wind field derived from all CRI beams with sufficient signal-to-noise ratio (SNR), the accuracy of the wind estimates for various DBS configurations is statistically analyzed. It is shown that for a three-beam DBS configuration, although the validity of the homogeneity assumption is enhanced at smaller zenith angles, the rootmean-square (RMS) error increases because of the ill-conditioned matrix in the DBS algorithm. As expected, inhomogeneities in the wind field produce large bias for the three-beam DBS configuration for large zenith angles. An optimal zenith angle, in terms of RMS error, of approximately $9^{\circ}-10^{\circ}$ was estimated. It is further shown that RMS error can be significantly reduced by increasing the number of off-vertical beams used for the DBS processing.
\end{abstract}

\section{Introduction}

Very high-frequency (VHF) and ultrahigh-frequency (UHF) profiling radars have been used widely in both the operational and research arenas for observations of the structure and dynamics of the atmosphere. In particular, this type of radar has proven important for studies of turbulence, momentum fluxes, and gravity waves (e.g., Röttger and Larsen 1990; Gage 1990; and references therein). One of the more common techniques for obtaining profiles of the three-dimensional wind field is the Doppler beam-swinging (DBS) method,

Corresponding author address: Dr. Boon Leng Cheong, School of Meteorology, University of Oklahoma, 120 David L. Boren Blvd., Suite 5900, Norman, OK 73072.

E-mail: boonleng@ou.edu where the antenna beam is sequentially steered between one vertical direction and two or more off-zenith directions at approximately $10^{\circ}-20^{\circ}$ (e.g., Strauch et al. 1984; Röttger and Larsen 1990). The vertical velocity can be obtained directly from the vertically pointing beam and can be used to improve the horizontal wind estimates (Strauch et al. 1987). Typically, the rather strict assumption that the three wind components (zonal, meridional, and vertical) are constant across the region defined by the beam locations is imposed. Mathematically, the spatial homogeneity assumption can be expressed as

$$
\mathrm{E}\left[\mathbf{v}\left(\mathbf{X}_{m}\right)\right]=\mathrm{E}\left[\mathbf{v}\left(\mathbf{X}_{n}\right)\right],
$$

where $\mathrm{E}$ represents the expected value operator and $\mathbf{v}$ represents the velocity vector at arbitrary positions $\mathbf{X}_{m}$ and $\mathbf{X}_{n}$ within the field of view. The accuracy and pre- 
cision (bias and variance) of the DBS wind measurements depends on several factors, such as the number of beams, zenith angles of the off-vertical beams, and the underlying atmospheric characteristics (Koscielny et al. 1984; Strauch et al. 1987). Bias of the wind estimates can be caused by a spatial gradient of the wind field, such as nonuniform vertical velocities across the observed region and/or horizontal shear (Koscielny et al. 1984). If any of these conditions occur, of course, the horizontal homogeneity assumption is no longer valid. Koscielny et al. (1984) have shown that the bias increases with increasing zenith angles of the off-vertical beams. On the other hand, the variance of DBS wind estimates, which may be due to the nonuniform reflectivity, turbulence, or signal-to-noise ratio (SNR), is reduced for large zenith angles. Nevertheless, an optimal zenith angle is generally difficult to obtain (Röttger and Larsen 1990). DBS measurements have shown good agreement with winds measured by other instruments, such as rawinsondes, towers, aircrafts, and other radar profiling techniques (e.g., see Röttger and Larsen 1990, and references therein). Recently, Adachi et al. (2005) reported that a five-beam DBS configuration can provide more accurate wind measurements compared to a three-beam configuration, especially if small-scale variability of vertical velocity is present. In general, the assumption of homogeneity is difficult to validate for each DBS measurement, although it can be examined statistically using a large amount of data (Strauch et al. 1987). In this work, an imaging radar is proposed to systematically investigate the accuracy of DBS wind measurements with various configurations of different beam separations and different numbers of beams. The goal is to demonstrate the type of analyses that can be performed using an imaging radar in a manner similar to conventional DBS-based radars. It should be noted that, for this study, the accuracy and precision of interest is not absolute, but is relative to the optimal measurements provided by the imaging radar.

Coherent radar imaging (CRI) was developed to reconstruct a two-dimensional, angular distribution of the atmospheric structure within the volume illuminated by the transmitted beam (e.g., Kudeki and Sürücü 1991; Palmer et al. 1998; Yu et al. 2000; Hélal et al. 2001). In addition, the angular distribution of radial velocity can be obtained by an efficient algorithm (Cheong et al. 2004). CRI can be thought of as a beam-forming technique, in which a number of receiving beams can be synthesized simultaneously by coherently combining signals from spatially separated receivers. As a result, an instantaneous snapshot of the power and radial velocity distributions is obtained within each resolution volume. Note that the synthesized beam is formed in software after the data are collected and, therefore, the beam directions and the number of beams are totally flexible. Of course, the independence of closely spaced beams depends on the overall aperture of the array and the algorithm used for processing. Nevertheless, this capability offers an unprecedented opportunity for the study of the accuracy and precision of the wind estimates from the DBS method. In this study, we focus on two unique features important for wind profiling radars: First, the horizontal distribution of reflectivity and radial velocity across the scanned DBS region are available using CRI and will be exploited to validate the uniform wind assumption. Second, various DBS configurations with different number of beams and pointing directions can be readily implemented using CRI with the exact same data, thus, eliminating concerns such as temporal separation and statistical stationarity.

In this work, the turbulent eddy profiler (TEP), developed by the University of Massachusetts (Mead et al. 1998), is used. TEP is a unique $915-\mathrm{MHz}$ boundary layer imaging radar with an array of up to 64 independent receivers. Using adaptive array processing, threedimensional volumetric images can be constructed within the field of view with a temporal resolution of approximately 2.5 s (Lopez-Dekker and Frasier 2004; Cheong et al. 2004). In addition to imaging clear-air turbulence, the TEP radar has been used for studies of the interaction of precipitation with turbulence (Palmer et al. 2005). Using adaptive methods, CRI also holds promise for the mitigation of both ground and biological clutter contamination (Cheong et al. 2006). For the current study, data collected on 15 June 2003 are processed using CRI to demonstrate the analysis of DBS accuracy in relation to the underlying wind field. This is the only applicable dataset that was collected during our field experiment in 2003. From the closest radiosonde station KALB, which is located approximately $110 \mathrm{~km}$ west of Amherst, Massachusetts, it is reported that the temperature was $22.8^{\circ} \mathrm{C}$, relative humidity was $61 \%$, and there was a northerly wind from $300^{\circ}$ at 11 $\mathrm{m} \mathrm{s}^{-1}$ during the experiment time.

The remainder of this article is organized as follows. A brief description of the TEP radar and experimental setup will be presented in section 2 , and experimental results will be presented in section 3 , followed by conclusions in section 4 .

\section{Experimental configuration}

The TEP radar consists of a corrugated horn antenna for transmission and an array of up to 64 microstrip patch elements for reception. The separation between receiving elements is approximately $0.57 \mathrm{~m}$ (Mead et al. 
1998; Lopez-Dekker and Frasier 2004). Analog signals from each receiver are sent through a low-noise amplifier and, subsequently, are downconverted to produce in-phase and quadrature components, which are digitized and stored for offline processing. For imaging radar applications, the transmitted beam is often relatively wide to provide a large field of view. TEP uses a separated horn antenna for transmission with a beamwidth of $25^{\circ}$. Narrowly focused receiving beams are synthesized simultaneously within the field of view using beam-forming/CRI techniques. As a result, a snapshot of the angular distribution of the atmosphere is obtained. This process can be conducted for each range gate independently to construct a three-dimensional volumetric image.

Because of the flexible and simultaneous beams provided through CRI, the TEP radar is ideal for the study of various DBS configurations with different pointing angles and numbers of beams. In addition, images of echo power and radial velocity across the region of across the DBS beams are obtained through the socalled pulse-pair beam-forming (PPB) process after successful phase calibration (Cheong et al. 2004). The PPB is an efficient joint implementation of beam forming and the pulse-pair processor, which can be used to estimate the spectral moments without the need of synthesizing time series for each beam position. In this work, the beams are formed in a $1^{\circ}$ increments within the transmit beam $\left(25^{\circ}\right.$ ), resulting in 490 pixels (or beam positions) in each image. Note that the effective angular resolution for the TEP radar using Fourier beam forming is approximately $3.5^{\circ}$. In this work, Capon (1969)-based adaptive PPB is implemented and an improved resolution of approximately $1.5^{\circ}$ is expected. Various DBS configurations can be obtained by selecting the desired beams (usually less than five) from the 490 synthesized directions. For beam-forming applications, a concern exists that the effective beam direction is skewed toward the center of the transmit beam, resulting in biased radial velocity estimates. However, Cheong et al. (2004) have shown that although the Capon PPB is optimized for power estimates, it also mitigates any biases in the radial velocity estimates caused by the transmit beam. Thus, only those radial velocities from Capon PPB are used in the present study.

As a reference wind field for this study, radial velocities from the 490 synthesized beams are used in a set of overdetermined equations to estimate the most representative, three-dimensional wind field over the imaged region; by "the most representative," we mean the least squares fit of a $3 \mathrm{D}$ wind vector to the radial velocity field within the region of interest, that is, covered by the 490 beams. This set of equations can be constructed as shown below (Palmer et al. 1993):

$$
\mathbf{C u}=\mathbf{v}_{r} \text {, }
$$

where

$$
\mathbf{C}=\left[\begin{array}{ccc}
\sin \theta_{1} \sin \phi_{1} & \sin \theta_{1} \cos \phi_{1} & \cos \theta_{1} \\
\sin \theta_{2} \sin \phi_{2} & \sin \theta_{2} \cos \phi_{2} & \cos \theta_{2} \\
\sin \theta_{3} \sin \phi_{3} & \sin \theta_{3} \cos \phi_{3} & \cos \theta_{3} \\
\vdots & \vdots & \vdots \\
\sin \theta_{m} \sin \phi_{m} & \sin \theta_{m} \cos \phi_{m} & \cos \theta_{m}
\end{array}\right], \quad \mathbf{u}=\left[\begin{array}{c}
U \\
V \\
W
\end{array}\right], \quad \mathbf{v}_{r}=\left[\begin{array}{c}
v_{r}^{(1)} \\
v_{r}^{(2)} \\
v_{r}^{(3)} \\
\vdots \\
v_{r}^{(m)}
\end{array}\right],
$$

and $\theta_{i}$ and $\phi_{i}$ are the zenith and azimuth angles of the $i$ th beam position, respectively. It is assumed that the wind field is uniform within the field of view defined by the transmit beam. The minimum least squares solution of the three-dimensional wind vector is obtained by the following equation:

$$
\mathbf{u}=\left(\mathbf{C}^{\mathrm{T}} \mathbf{C}\right)^{-1} \mathbf{C}^{\mathrm{T}} \mathbf{v}_{r},
$$

where the $(\cdot)^{\mathrm{T}}$ is the transpose operator. In this work, radial velocity estimates from 490 beams were generated and several hundreds of them with SNR larger than $5 \mathrm{~dB}$ were used to estimate the wind field. This three-dimensional wind field estimate derived from this overdetermined set of equations will be considered the reference for the latter comparisons, given that it represents the average uniform wind field (in the least squares error sense) over the view of the TEP. One of the goals of this work is to investigate how well the wind estimates obtained from a conventional DBS system with three to five beam positions represents the spatially averaged wind for various configurations.

The data were collected by the TEP with 61 independent receivers in Amherst on 15 June 2003. A 222-ns pulse was used with range resolution of $33.3 \mathrm{~m}$. Using a pulse repetition frequency (PRF) of $35000 \mathrm{~Hz}$ and 250 coherent integration, the aliasing velocity is $11.5 \mathrm{~m} \mathrm{~s}^{-1}$ 


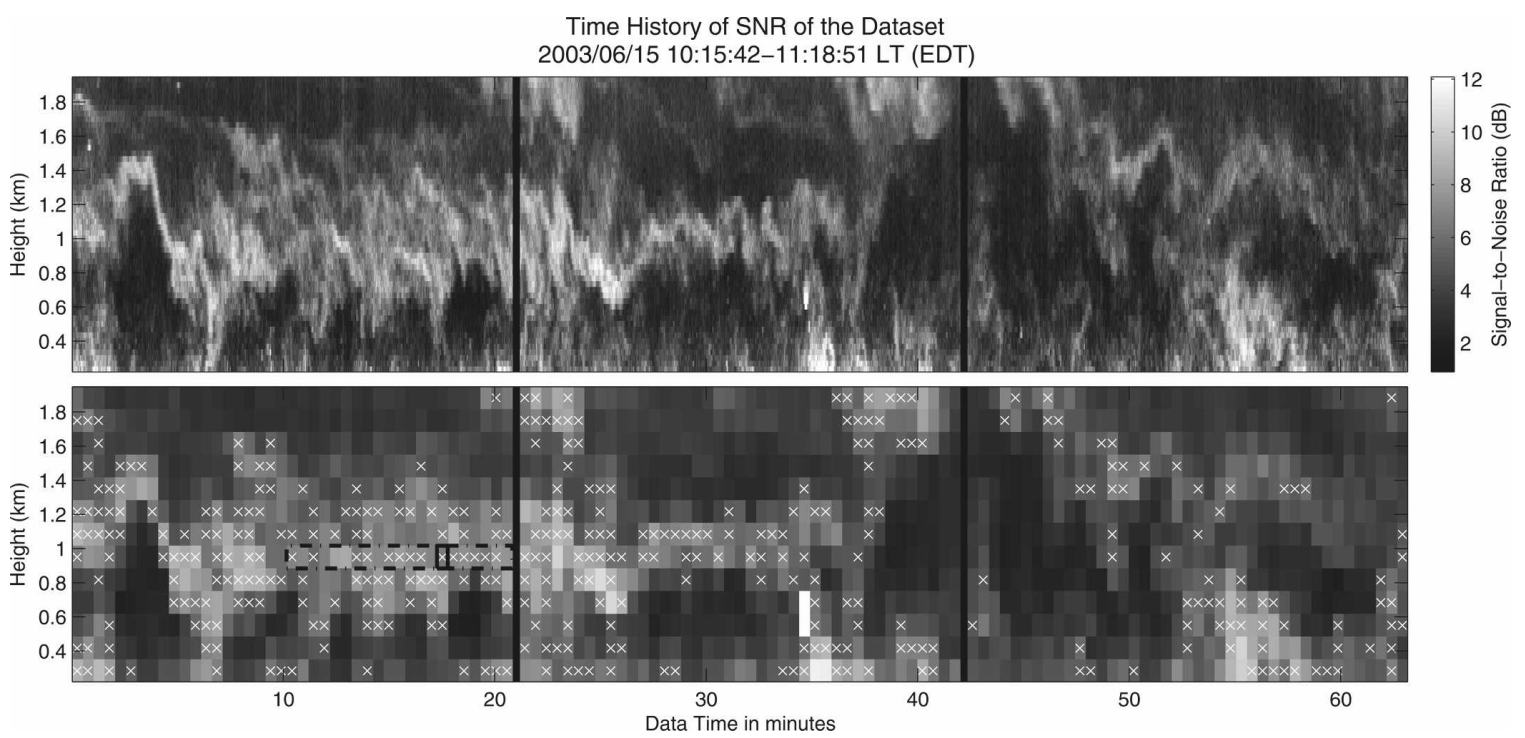

FIG. 1. During the 63-min period, vigorous convective and mixing processes can be observed within the boundary layer. The boxed regions denote regions of further study, which will be investigated in the later portions of this work.

given the effective interpulse period (IPP) of $7.14 \mathrm{~ms}$. Each record of raw time series data has 260 points and is used for CRI processing. There is a 0.64-s gap between each 260-pulse dwell packet for processing overhead. As a result, the temporal resolution is $2.5 \mathrm{~s}$. The top panel in Fig. 1 shows the height-time intensity of SNR from the center beam of the TEP radar in the summer morning of 2003 from 1015 to 1118 LT, with sunrise at approximately 0600 LT. The bottom panel shows the same dataset, but with a more coarse resolution. The resultant temporal and range resolution are $30 \mathrm{~s}$ and $133 \mathrm{~m}$ after incoherently averaging 12 records of data and four range gates, respectively, in order to improve spatial homogeneity. The boxed regions indicate time and heights where the wind measurements will be investigated in the following section.

\section{Experimental results}

A subset of the data, as emphasized by a dashed-line box in the lower panel of Fig. 1, was extracted and the dataset for CRI was further averaged over a 10-min period to obtain the spatial homogeneity of the wind field. The averaging period was determined from a trial-and-error procedure until homogeneous wind field (parallel contours for the radial velocity) maps were found. Images of the resultant SNR and radial velocity distributions are shown in the upper-left and upperright panels of Figs. 2a,b, respectively. Although the TEP is not calibrated for absolute reflectivity, an SNR map can still provide a good approximation of the dis- tribution of relative reflectivity. The reference horizontal wind vector derived from the overdetermined Eq. (3) is depicted by a thick arrow on the radial velocity map. In this case, the reference horizontal wind speed is approximately $7.38 \mathrm{~m} \mathrm{~s}^{-1}$ and the vertical velocity is negligible. Two adjacent contour lines encompassing zero radial velocity are denoted by thin lines. It is clear that although the reflectivity is not homogenous the wind field is approximately uniform, which is manifested by parallel radial velocity contours that are perpendicular to the wind direction. The conventional three-beam DBS system, used for this study, samples three locations of the atmosphere with one vertical beam and two off-zenith beams. These beam directions are depicted by small circles in Fig. 2c with a zenith angle of $10^{\circ}$ for both off-vertical beams. The cross sign represents the angular rotation of the three-beam system. By rotating the off-vertical beams in azimuth, different regions of the atmosphere with the same zenith are sampled. If the assumption of uniform wind is valid, DBS wind estimates from different azimuth angles would be similar given that the SNR from the three beam directions are sufficiently high. This testing scheme is demonstrated Fig. 2d. The abscissa represents the angular rotation of the three-beam DBS configuration in which positive angles indicate clockwise rotation. The RMS difference between the three-beam DBS wind estimates and the reference is calculated over the $360^{\circ}$ span of azimuth angles. These errors are denoted in the figure for the zonal $(U)$ and meridional $(V)$ components from left to right, respectively. It is 

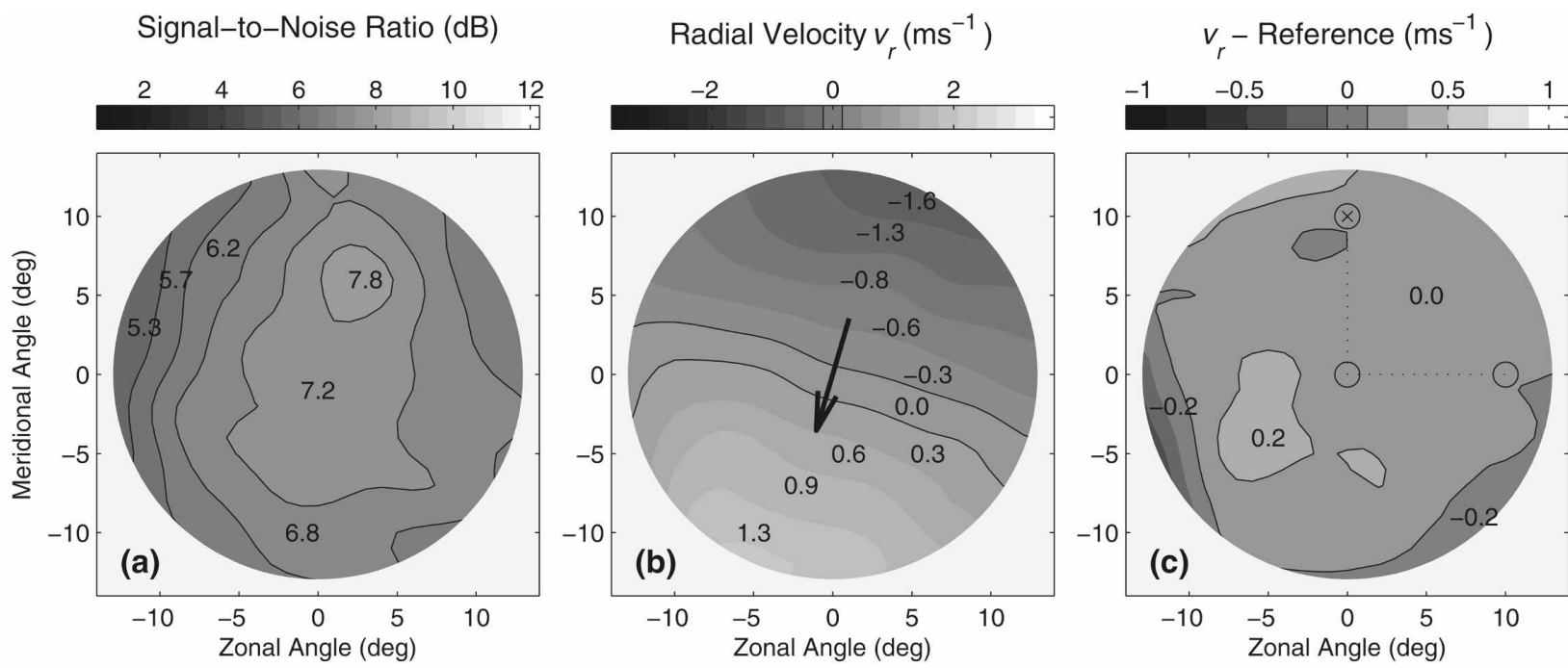

Different Estimates from 3-beam DBS

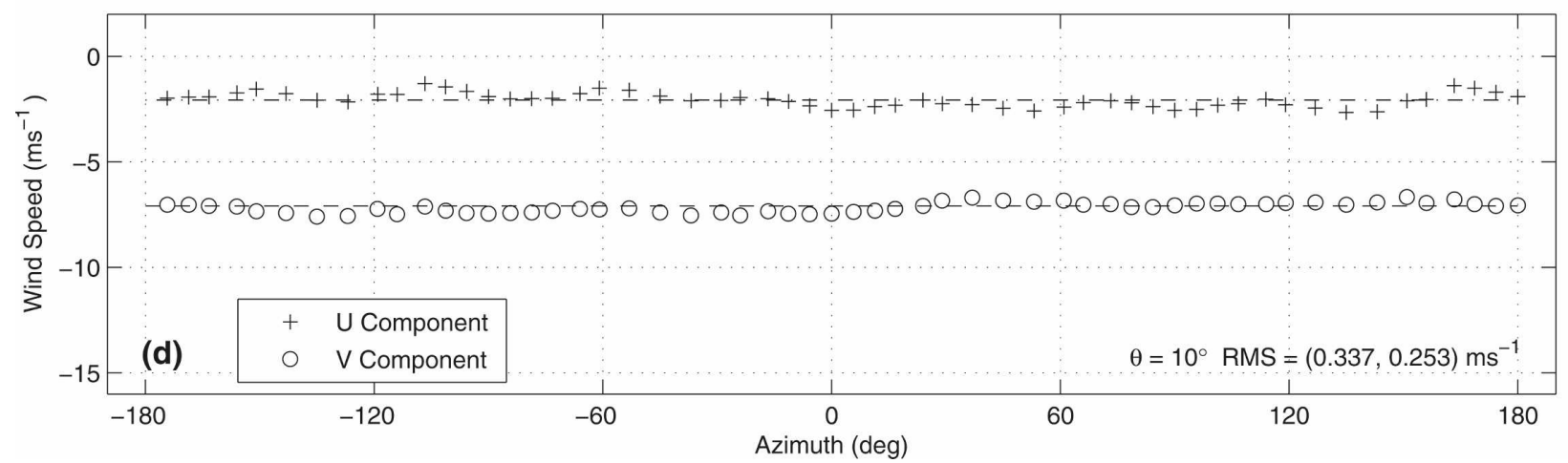

FIG. 2. Maps of (a) SNR and (b) radial velocity, and (c) the difference from the reference within the field of view are provided here. After a 10-min average, the SNR map does not exhibit rapid spatial variability as would be expected for a shorter time average. Furthermore, the estimated radial velocity field exhibits a set of near-parallel contours, which is expected from a nearly homogeneous wind field. In this case, the average wind speed is approximately $7.38 \mathrm{~m} \mathrm{~s}^{-1}$. The three circles in (c) indicate the beam positions $\left(10^{\circ}\right.$ zenith angle) used for the DBS configuration. The cross signs represent the angular rotation of the three-beam system. (d) By rotating the two off-vertical beams over $360^{\circ}$ in azimuth angle, the RMS error, relative to the overdetermined reference case shown in Eq. (3), was calculated and shown. The dashed line indicates the average wind components derived from the reference wind. By examining difference between the three-beam DBS wind estimates and the reference wind field, the uniform wind field assumption can be tested.

evident that the RMS error for all three wind components is relatively small for this case of a 10 -min average, where the homogeneity assumption is likely to be valid.

The same analysis is performed on data with a shorter dwell time of $30 \mathrm{~s}$. Figure 3 shows the results from the smaller region denoted by the solid box in the lower panel of Fig. 1. The reference wind is obtained using the same short dwell time and is slightly different from the one from previous example. As expected, small-scale reflectivity variations are more evident for short dwell times. For example, significantly lower $\mathrm{SNR}$, that is, more than $3 \mathrm{~dB}$ below the peak, can be observed in the western portion of the map. In addition, two distinct, high-powered "blobs" centered at meridional angles of $5^{\circ}$ and $-6^{\circ}$ and a zonal angle of $5^{\circ}$ are observed. Using standard Fourier-based beam forming, such blobs have been observed to cause significant errors in wind field estimates (Cheong et al. 2004). However, Capon-based beam forming, as used for this study, significantly reduces any such errors by adapting the received beam pattern to the existing power structure. More important than the SNR structure, the contours of radial velocity for this 30 -s average do not exhibit homogeneous characteristics (Figs. 3b,c) in comparison to those shown in Fig. 2, for the 10-min average. In other words, the spatial variability of the threedimensional wind field is enhanced if shorter time av- 

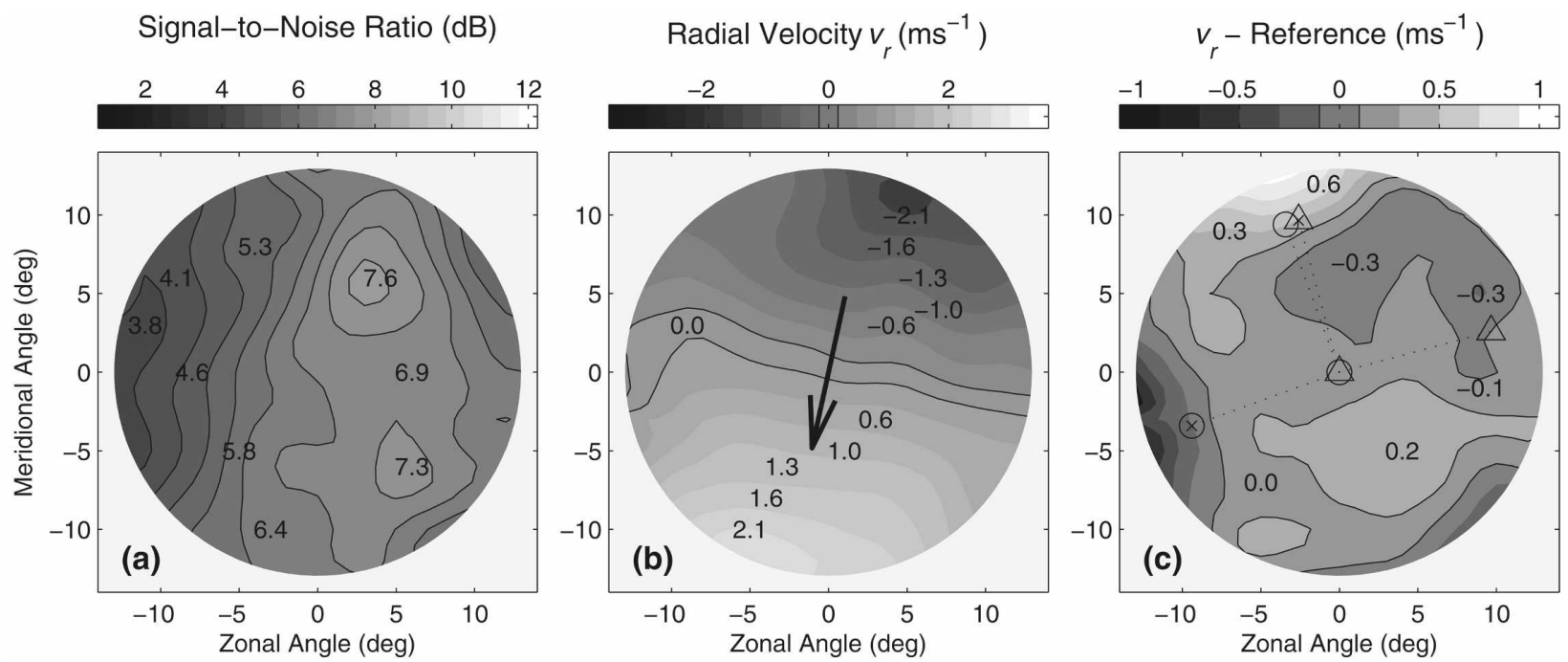

Different Estimates from 3-beam DBS

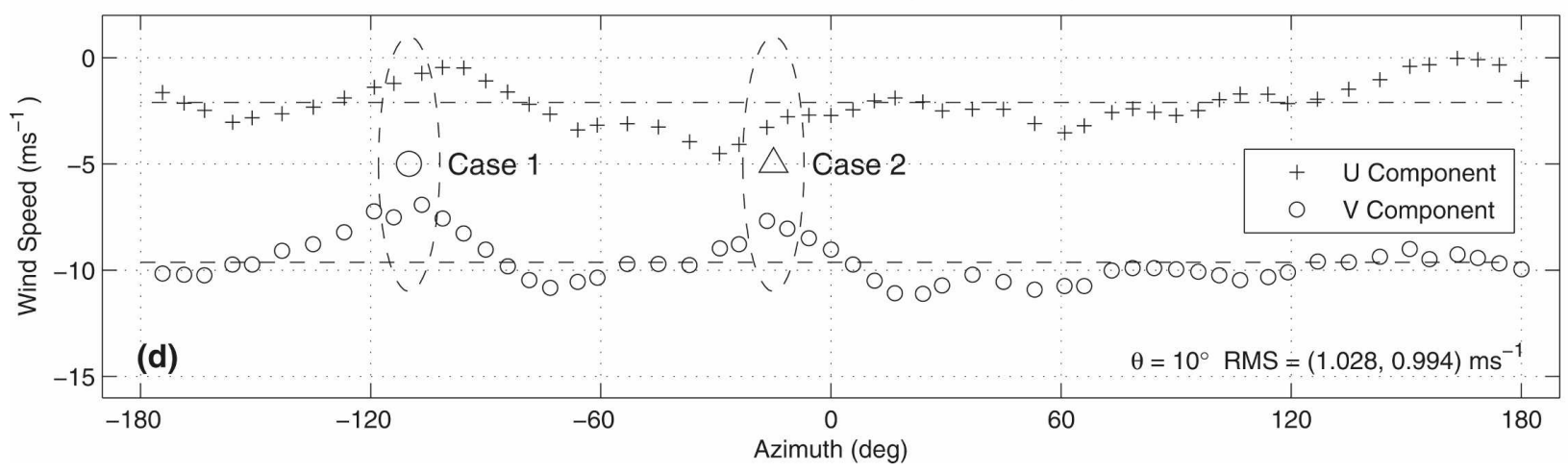

FIG. 3. Same as in Fig. 2, but for a 30-s average; in this example, the atmospheric structure is less homogeneous as seen in the spatial structure of the wind field.

erages are used. As a result, the assumption of uniform wind is no longer valid. Therefore, the wind fields derived from the three-beam DBS configuration can be significantly biased if one or more beam directions are located in nonuniform wind regions.

For further study, two specific cases with large bias in the $V$ component are selected and are denoted in Fig. 3 as case 1 (azimuth angle of $-115^{\circ}$ ) and case 2 (azimuth angle of $-18^{\circ}$ ). The corresponding beam directions for cases 1 and 2 are emphasized in Fig. $3 c$ by circles and triangles, respectively. It is interesting to note that for these two cases, one of the off-vertical beams is coincident and is located near the most significant deviation of the radial velocity locating almost north $\left(350^{\circ}\right)$, which can explain the two large biases in the $V$ component (cases 1 and 2). In contrast, smaller bias is observed if the off-vertical beams are directed away from this nonuniform region, that is, azimuth angles of approximately $30^{\circ}-180^{\circ}$. Moreover, the general RMS error from the 30 -s-averaged data is larger than for the 10-min average, as expected. As is generally understood, nonuniformity in the wind field can bias the DBS technique (Koscielny et al. 1984; Strauch et al. 1987).

The next obvious question is whether the effects of wind field inhomogeneities can be mitigated by using smaller zenith angles in a DBS configuration. Given the flexibility of an imaging radar, this question is now investigated by repeating the previous three-beam DBS experiment but for different zenith angles $(\theta)$. For the same data shown in Fig. 3, the RMS error (over all azimuth angles) was calculated for the three wind components as a function of zenith angle. To more clearly demonstrate the trend of RMS as a function of zenith angle, each curve in the top panel of Fig. 4 was normalized to a minimum of zero with the resultant RMS defined as relative RMS. The absolute mean and standard deviation of the RMS trend is shown as an error bar plot in the middle panel. The histogram of the zenith angle where the minimum RMS error occurs from all curves in the upper panel is shown in the bottom panel. 

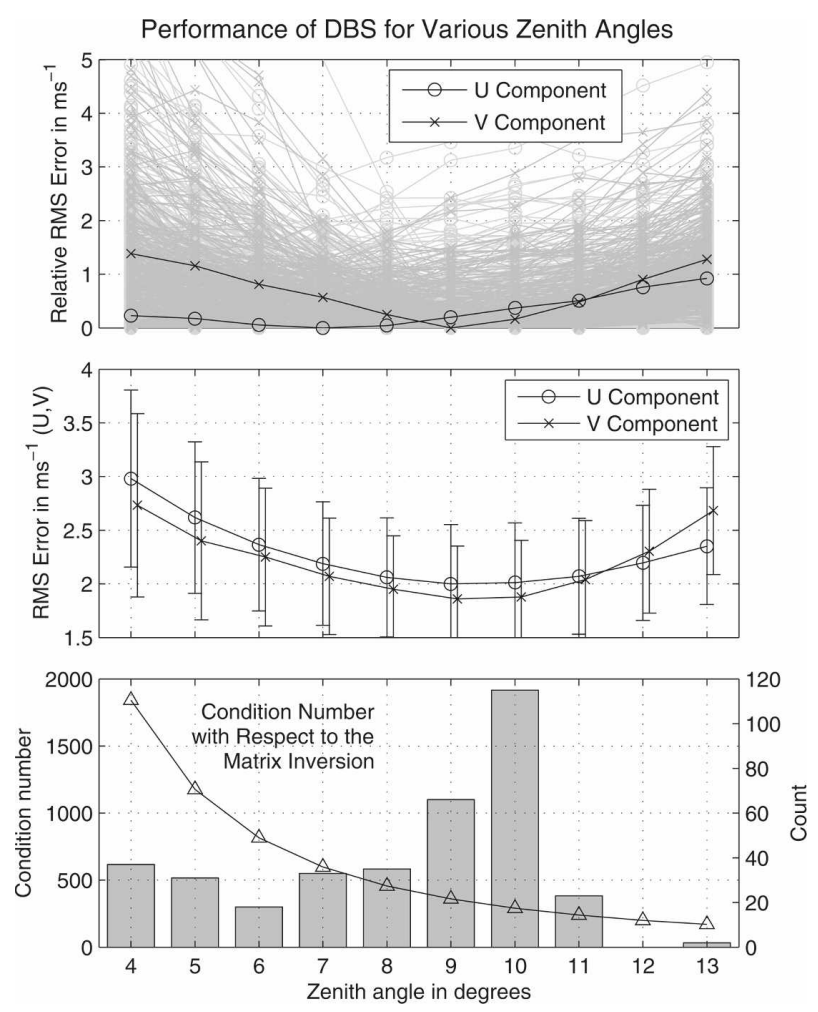

FIG. 4. A collection of relative RMS error from approximately 360 samples as a function of the zenith angle is shown to illustrate the quantification of spatial homogeneity assumption. (top) The dark solid lines are the results from the data shown in Fig. 3. (middle) The absolute mean and standard deviation of all cases are shown as error bars to show the precision and average bias of DBS-derived wind vectors (from the reference). (bottom) The histogram of the zenith angle of minimum RMS difference is presented. Slightly over $50 \%$ of the data show a minimum RMS occurring at approximately $9^{\circ}-10^{\circ}$, which may be a result of different sources dominating the accuracy of the DBS wind measurements at small and large zenith angles. The condition number with respect to the matrix inversion in Eq. (3) is also shown.

It is clear from these results that the three-beam DBS has predominant $(50 \%)$ minima at zenith angles from $9^{\circ}$ to $10^{\circ}$. Note that the RMS error is defined as a measure of the azimuthal average of the deviation of the DBS winds from the reference. Moreover, the reference is the uniform wind field that best fits with the radial velocity distribution obtained by the imaging radar. It is interesting that the RMS error of $U$ and $V$ components generally decreases with increasing zenith angle until approximately $\theta=7^{\circ}$ and $\theta=9^{\circ}$, respectively, although this effect is more prominent for the $V$ component. Beyond this zenith angle, the RMS error of both components increases along with zenith angle. A similar trend between the zenith angles of $4^{\circ}$ and $13^{\circ}$ is observed from the other numerous cases whose results are denoted by light lines in the figure. Those cases, depicted by cross signs in Fig. 1, are selected from regions where SNR is sufficiently high $(>5 \mathrm{~dB})$ and the variability of radial velocity distribution is relatively large. Among these cases, the majority share similar properties with the special example just discussed in which convective processes were vigorous within the boundary layer.

Many factors can contribute to the observed RMS error, such as statistical uncertainty in the radial velocity measurements, spatial variability of the wind components, the number of beams, and zenith angle. For short dwell times, it was demonstrated in Fig. 3 how spatial inhomogeneity could affect the resulting wind field from DBS. Koscielny et al. (1984) have shown that the bias of wind measurements increases with zenith angle for a linear wind field for a given height. This could be the dominant factor that causes the RMS error to increase with zenith angle at large beam separation $\left(\theta\right.$ is larger than approximately $9^{\circ}$ ). For small zenith angles, however, another possible cause of error is an increase in statistical variance of the wind estimate (Koscielny et al. 1984). In other words, even though the assumption of uniform wind is more likely satisfied at smaller zenith angles, the variance of the wind estimate becomes larger and can still degrade the results. Another way to understand this statistical error is through the inversion of the $\mathbf{C}$ matrix in Eq. (2). At smaller zenith angles, the inversion is more susceptible to errors in pointing direction and lack of independence of radial velocity estimates. These effects are manifested in the condition number of $\mathbf{C}$, which is shown in the bottom panel of Fig. 4 for several different zenith angles. A larger condition number indicates the matrix is closer to singularity. Although it is assumed that the errors in effective beam direction from the Capon beam forming are negligible, because of the adaptive nature of the algorithm, in practice small bias can still be present. As a result, additional error can be introduced to the inversion of the $\mathbf{C}$ matrix (e.g., Thorsen et al. 1997). The effect could become more severe at smaller zenith angles where larger condition number occurs. If the true bias can be determined, it is possible to characterize the supremum of error using the condition number, but for our case this is prohibited.

In contrast to the argument of smaller zenith angle resulting in larger error, other scenarios could result in minimum RMS error at smaller zenith angles. For example, it is possible that the wind field could have small-scale spatial variability that dominates the RMS. In other words, the RMS difference in this case is a measure of the bias that increases with increasing zenith angle. Nevertheless, the existence of minimum RMS within $4^{\circ}$ and $13^{\circ}$ implies that an optimal DBS 


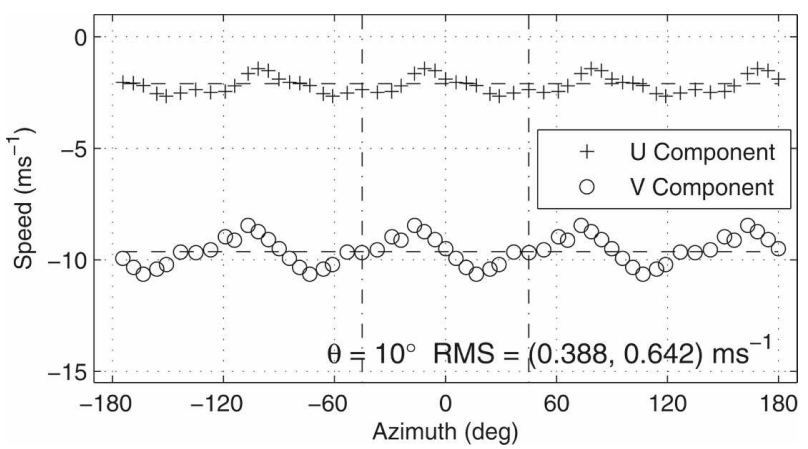

FIG. 5. Same as in Fig. 3 (bottom), but for a five-beam DBS configuration. For these directions, it is expected that the results will be periodic every $90^{\circ}$, in which a period is indicated by the dashed-dotted lines at $\pm 90^{\circ}$.

configuration can be obtained despite the competing factors we just discussed, that is, small beam separation to satisfy spatial homogeneity assumption versus large beam separation to improve the statistical property of matrix inversion. However, the determination of optimal beam configurations under all conditions is an extremely challenging problem.

Another obvious mitigation scheme for the spatial inhomogeneity problem would be to use more than three beam positions. The same data shown in Fig. 3 are now used to simulate a five-beam DBS configuration in which two more beams are added in the opposite directions of the previous two off-vertical beams. An example for $\theta=10^{\circ}$ with different regions of the atmosphere being sampled is shown in Fig. 5. As one would expect, the variability of the five-beam DBS wind as a function of azimuth angle is smaller than for the threebeam DBS case. Obviously, this results from the fact that additional, independent information is introduced to Eq. (2) by the addition of the new beam directions. Note that the resultant wind vectors are periodic every $90^{\circ}$ because the five-beam constellation becomes identical to itself with every $90^{\circ}$ rotation. Furthermore, a nine-beam configuration, with eight evenly spaced positions in azimuth and one vertical beam, is produced with the result presented in Fig. 6. The RMS error is even smaller in this case because the configuration is closer to the one used for reference wind estimation. In addition, the periodicity of the RMS error becomes $45^{\circ}$, as specified by the two dashed vertical lines.

Although many of results presented here are relatively obvious to experienced researchers in the wind profiling community, these data represent the first use of an imaging radar to investigate the effects of wind field inhomogeneities on the DBS method. By no other means is it possible to simultaneously produce results from many different DBS configurations and use these

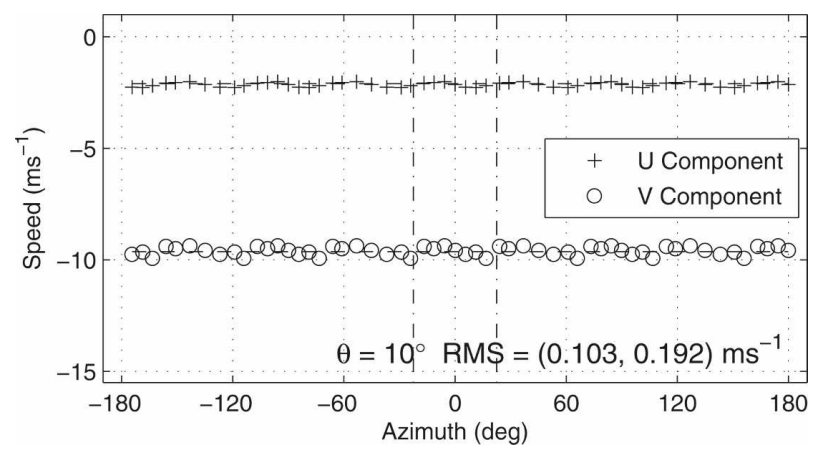

FIG. 6. Similar to Fig. 5, but for a nine-beam DBS configuration.

for statistical analyses of their relative advantages and disadvantages.

\section{Conclusions}

In the present work, the use of an imaging Doppler radar for the study of wind field inhomogeneity effects on standard DBS measurements has been examined. By design, CRI techniques provide the power and flexibility to be able to view the structure and dynamics of the atmosphere within the pulse volume defined by the transmit beam. If a relatively wide transmit beam is used, it is possible to simultaneously image hundreds of locations where only a few are typically used for conventional DBS. By selecting the desired subset of beams from the imaged beam set, the present study has provided a statistical comparison of an unprecedented number of DBS configurations with varying zenith and azimuth angles.

The particular imaging radar used for this study is the TEP. Data of an experimental campaign from June 2003 (Cheong et al. 2004; Palmer et al. 2005) were used for this investigation. Using Capon imaging (Palmer et al. 1998), the TEP data were processed to produce highresolution maps of SNR and radial velocity for both a 10-min and 30-s average. By comparing the results from these two averaging periods, it was possible to show that the horizontal homogeneity assumption was valid for $10 \mathrm{~min}$, but not for 30 -s data. Using the 10-min average results as the reference for both the 10-min and 30-s dwell studies, RMS error was calculated for numerous beam configurations. It was shown that for a relatively short dwell time average (30 s), the particular choice of azimuthal sampling was exceedingly important for the performance of DBS. By examining the radial velocity map, it was evident that wind field inhomogeneities caused the observed bias and variability. Reducing the zenith angle of the DBS configuration 
minimized the area defined by the DBS scanning pattern and thus enhanced the validity of the homogeneity assumption. However, extremely small zenith angles $\left(4^{\circ}\right)$ also produced erroneous results primarily due to the near singularity of the matrix involved in the DBS processing. These trade-offs resulted in an optimal zenith angle choice of approximately $9^{\circ}-10^{\circ}$. Finally, an obvious solution to the homogeneity problem was examined by creating DBS configurations with larger number of off-zenith beam positions. As expected, both five- and nine-beam configurations reduced the bias and variance resulting from deviations from the uniform wind field assumption.

Through the use of CRI techniques, a statistical analysis has been performed without the experimental uncertainties typically present when multiple radar configurations must be implemented. In addition to the aforementioned outcome of this analysis, one of the goals of this work is to emphasize to the profiling radar community the breadth of problems that can be studied using imaging techniques. Radars, such as TEP, hold promise for many high-resolution applications and should be explored for future research.

Acknowledgments. BLC, RDP, and MWH were supported by the Army Research Office through Grant DDAD19-01-1-0407. SJF and FJL-D were supported by the Army Research Office (Atmospheric Sciences) through Grant DAAG55-98-1-0480.

\section{REFERENCES}

Adachi, A., T. Kobayashi, K. S. Gage, D. A. Carter, L. M. Hartten, W. L. Clark, and M. Fukuda, 2005: Evaluation of threebeam and four-beam profiler wind measurement techniques using a five-beam wind profiler and collocated meteorological tower. J. Atmos. Oceanic Technol., 22, 1167-1180.

Capon, J., 1969: High-resolution frequency-wavenumber spectrum analysis. Proc. IEEE, 57, 1408-1419.

Cheong, B. L., M. W. Hoffman, R. D. Palmer, S. J. Frasier, and F. J. López-Dekker, 2004: Pulse pair beamforming and the effects of reflectivity field variations on imaging radars. $R a$ dio Sci., 39, RS3014, doi:10.1029/2002RS002843.

,,,$--- \ldots$, and $\_, 2006$ : Phase-array design for bio- logical clutter rejection: Simulation and experimental validation. J. Atmos. Oceanic Technol., 23, 585-598.

Gage, K. S., 1990: Radar observations of the free atmosphere: Structure and dynamics. Radar in Meteorology: Battan Memorial and 40th Anniversary Radar Meteorology Conference, D. Atlas, Ed., Amer. Meteor. Soc., 534-565.

Hélal, D., M. Crochet, H. Luce, and E. Spano, 2001: Radar imaging and high-resolution array processing applied to a classical VHF-ST profiler. J. Atmos. Solar-Terr. Phys., 63, 263 274.

Koscielny, A. J., R. J. Doviak, and D. S. Zrnić, 1984: An evaluation of the accuracy of some radar wind profiling techniques. J. Atmos. Oceanic Technol., 1, 309-320.

Kudeki, E., and F. Sürücü, 1991: Radar interferometric imaging of field-aligned plasma irregularities in the equatorial electrojet. Geophys. Res. Lett., 18, 41-44.

Lopez-Dekker, P., and S. J. Frasier, 2004: Radio acoustic sounding with a UHF volume imaging radar. J. Atmos. Oceanic Technol., 21, 766-776.

Mead, J. B., G. Hopcraft, S. J. Frasier, B. D. Pollard, C. D. Cherry, D. H. Schaubert, and R. E. McIntosh, 1998: A volume-imaging radar wind profiler for atmospheric boundary layer turbulence studies. J. Atmos. Oceanic Technol., 15, 849859.

Palmer, R. D., M. F. Larsen, and E. L. Sheppard, 1993: Poststatistic steering wind estimation in the troposphere and lower stratosphere. Radio Sci., 28, 261-271.

— S. Gopalam, T. Yu, and S. Fukao, 1998: Coherent radar imaging using Capon's method. Radio Sci., 33, 1585-1598.

, B. L. Cheong, M. W. Hoffman, S. J. Frasier, and F. J. LópezDekker, 2005: Observation of the small-scale variability or precipitation using an imaging radar. J. Atmos. Oceanic Technol., 22, 1122-1137.

Röttger, J., and M. F. Larsen, 1990: UHF/VHF radar techniques for atmospheric research and wind profiler applications. $R a-$ dar in Meteorology: Battan Memorial and 40th Anniversary Radar Meteorology Conference, D. Atlas, Ed., Amer. Meteor. Soc., 235-281.

Strauch, R. G., D. A. Merritt, K. P. Moran, K. B. Earnshaw, and D. V. Kamp, 1984: The Colorado wind-profiling network. $J$. Atmos. Oceanic Technol., 1, 37-49.

—, B. L. Weber, A. S. Frisch, C. G. Little, D. A. Merritt, K. P. Moran, and D. C. Welsh, 1987: The precision and relative accuracy of profiler wind measurements. J. Atmos. Oceanic Technol., 4, 563-571.

Thorsen, D., S. J. Franke, and E. Kudeki, 1997: A new approach to MF radar interferometry for estimating mean winds and momentum flux. Radio Sci., 32, 707-726.

Yu, T.-Y., R. D. Palmer, and D. L. Hysell, 2000: A simulation study of coherent radar imaging. Radio Sci., 35, 1129-1141. 